\title{
Come ridurre il rischio cardiovascolare attraverso il controllo del metabolismo calcio fosforo nell'uremico in dialisi, nel Terzo Millennio?
}

\author{
M. Marangella \\ S.C. Nefrologia e Dialisi, ASO Ordine Mauriziano, Torino
}

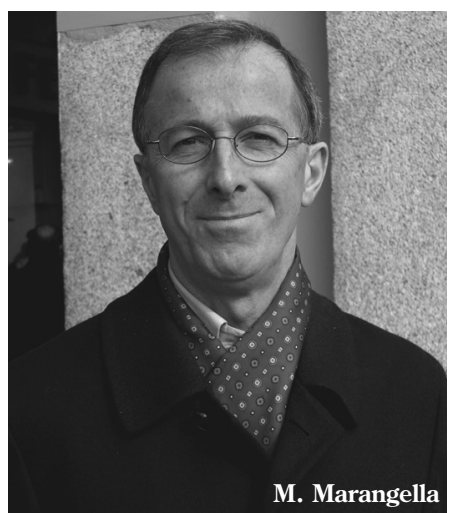

\section{Introduzione}

Il rischio cardiovascolare $(\mathrm{CV})$ è significativamente aumentato non solo nei pazienti in emodialisi ma anche in quelli con stadi meno avanzati di insufficienza renale.

I dati epidemiologici su cui si fonda questo assunto sono: 1) La mortalità $\mathrm{CV}$ è particolarmente elevata in emodialisi, e rende conto di circa il 50\% delle morti di questi pazienti (1 -3).

2) Nella stessa fascia d'età il rischio di morte per causa $\mathrm{CV}$ dei pazienti uremici in emodialisi è molto maggiore rispetto alla popolazione generale. La forbice è particolarmente ampia nei soggetti più giovani e tende a restringersi, senza annullarsi verso la VIII-IX decade (4).

3) Linsufficienza renale, fin dagli stadi iniziali, aumenta il rischio di morte da causa $\mathrm{CV}$ e di ospedalizzazione (5). Sembrerebbe pertanto che, nella insufficienza renale, alcuni fattori peculiari si aggiungano ai fattori di rischio classici, e tipici della popolazione generale. È stato per questo calcolato che ai fattori di rischio $\mathrm{CV}$ classici la peculiarità della uremia aggiunge un rischio relativo pari a 2.5 per linfiammazione, 1.5 per lo stress ossidativo, 1.4 per le alterazioni del metabolismo calcio-fosforo (1). Il concorso dei diversi fattori specifici per l'uremia deter- mina un rischio cumulativo, derivante dal moltiplicarsi dei singoli fattori, pari a circa 70-80. Dunque, in corso di uremia, il rischio $\mathrm{CV}$ è circa $70-80$ volte maggiore rispetto a quello della popolazione generale.

Fra i fattori specifici dell'uremia, solo alcuni sono modificabili con la terapia. Fra questi le alterazioni del metabolismo minerale sono quelle su cui il nefrologo è in grado di intervenire con una qualche efficacia.

Rispetto a questo, che sarà argomento del presente articolo, le problematiche che si pongono sono le seguenti: 1) Le alterazioni del metabolismo minerale hanno effetti sulla mortalità in generale e sul rischio $\mathrm{CV}$ nel paziente uremico?

2) Questo effetto se esiste, è quantizzabile?

3) Gli strumenti terapeutici e di prevenzione disponibili sono in grado di ridurre il danno CV?

Per motivi pratici conviene sviluppare l'argomento considerando singolarmente i seguenti componenti del metabolismo calcio fosforico:

1) Ruolo del PTH e della prevenzione dell'iperparatiroidismo secondario (S-HPT).

2) Prodotto calcio fosforo (CaxP): effetto su rischio $\mathrm{CV}$ e risultati della correzione delle sue alterazioni.

3) Vitamine D e rischio CV.

Ovviamente non si tratta di momenti fisiopatologici e terapeutici indipendenti, ma strettamente connessi e interagenti fra loro, in modo tale da porre spesso problematiche di gestione della terapia e di prevenzione di effetti indesiderati. 
Verranno di seguito trattati singolarmente, proponendo poi considerazioni finali in ordine all'ottimizzazione del trattamento, in particolare di quelli concernenti il rischio CV.

\section{Il PTH e iperparatiroidismo secondario}

Un citatissimo studio di Block evidenzia che, dal punto di vista epidemiologico, i pazienti in dialisi con PTH più elevato hanno un aumento del rischio di morte di circa il $20 \%$ per valori di PTH intatto $>600 \mathrm{pg} / \mathrm{mL}$ (2). Questo aumento del rischio è più sostenuto per valori di PTH al di sopra di 900-1200 pg/mL. Più importante appare la dipendenza della morte $\mathrm{CV}$ dai livelli di PTH, in quanto il rischio relativo risulta aumentato da 1 a 3.9 per ivelli di PTH maggiori di $476 \mathrm{pg} / \mathrm{mL}$, in uno studio epidemiologico condotto in Europa su 143 pazienti emodializzati, seguiti per 6 anni (6). Anche il rischio di morte improvvisa, spesso riferibile a causa cardiaca, era risultato moderatamente aumentato per valori di PTH $>495 \mathrm{pg} / \mathrm{mL}$ (1). Dati analoghi emergono rispetto al tasso di ospedalizzazione durante un follow-up di 12-18 mesi. Circa il 60\% dei pazienti dello studio hanno avuto un ricovero ospedaliero, nel $24.1 \%$ causa di patologia CV. Il rischio era significativamente più elevato nei pazienti con $\mathrm{PTH}>900$ $\mathrm{pg} / \mathrm{mL}(2)$.

Un altro studio recente analizza la capacità predittiva del rischio di morte dei parametri di metabolismo minerale, considerando sia i valori basali che quelli ripetuti trimestralmente nel corso di 2 anni (3). Pur non trattandosi di dati specifici per il rischio di morte $\mathrm{CV}$, dallo studio emergono indicazioni interessanti rispetto al ruolo del PTH. Si conferma un andamento $U$-shaped della relazione fra rischio di morte e livelli di PTH, sia basali sia di followup. L'aumento del rischio per valori $<150 \mathrm{pg} / \mathrm{mL}$ è significativo e si mantiene anche dopo aggiustamento per variabili confondenti, anagrafiche, biochimiche, di terapia e correlate allo stato nutrizionale. Sorprendente è l'andamento del rischio per valori elevati di PTH. Si osserva perfino una lieve riduzione del rischio (Hazard ratio < 1.0) per valori compresi fra 500 e $700 \mathrm{pg} / \mathrm{mL}$. Tuttavia questo andamento viene completamente sovvertito quando i dati siano corretti per le variabili confondenti. Infatti il rischio sale in modo lineare fino al $50 \%$ quando il PTH eccede $700 \mathrm{pg} / \mathrm{mL}$. Fra le variabili confondenti emerge evidente la terapia con vitamina $\mathrm{D}$, di cui sarà detto sotto (3).

In un lavoro molto recente è stata analizzata in modo retrospettivo la relazione fra mortalità e aderenza alle linee guida K-DOKI (7). Sono stati esaminati i dati dei primi 3 mesi di dialisi ottenuti da 13792 pazienti avviati al trattamento sostitutivo presso una agenzia non-profit (Dialysis Clinic Inc., DCI). La percentuale di pazienti in target rispetto al PTH si è mantenuta stabile intorno al 30\%. In questi il rischio di morte era sempre inferiore rispetto ai pazienti fuori target, con hazard ratio variabile fra 0.83 0.95 . Il vantaggio era più consistente nella fascia di età di 20-44 anni. Pur trattandosi di una semplificazione, è ragionevole prevedere che una consistente quota di questa mortalità fosse da causa $\mathrm{CV}$. Fra le altre variabili significative erano l'età, i livelli di PCR, albuminemia, fosforemia. Fra gli altri, l'assunzione di vitamina D per almeno 1 mese si associava a minor rischio di morte, sia pure con una bassa significatività. Più importante sembrava in questo il confronto fra sevelamer e chelanti contenenti calcio (7).

In una coorte di 127 pazienti avviati alla dialisi, i decessi a 18 mesi furono $26.7 \%$. I livelli basali di PTH, insieme ad altre variabili, erano predittivi di morte, mentre le medie dei valori in follow-up erano maggiori nei deceduti rispetto a quelli sopravvissuti (8).

Infine, un recente studio sperimentale condotto nei ratti uremici e sottoposti a PTX, ha dimostrato che l'infusione di PTH è causa di calcificazione della media aortica e di calcificazione coronarica, indipendentemente dall'andamento della fosforemia (9).

Il messaggio chiaro che sembra emergere dagli studi fin qui citati è che il mantenimento dei livelli di PTH fra 150 e $300 \mathrm{pg} / \mathrm{mL}$ si associa alla maggior protezione dal rischio $\mathrm{CV}$, confermando indirettamente che anche valori troppo bassi di PTH aumentano il rischio. Pertanto, i dati della letteratura giustificano ogni sforzo terapeutico volto a portare i valori di PTH entro il target consigliato dalle Linee Guida correnti.

Tuttavia, il fatto che tutti gli studi che abbiamo citato siano retrospettivi, riduce di molto la forza di questa raccomandazione. Pertanto non possiamo a oggi affermare in modo definitivo che gli strumenti terapeutici capaci di influenzare i livelli di PTH siano in grado di migliorare gli outcome CV del paziente uremico. I dati epidemiologici imputano a situazioni di PTH soppresso un aumento del tasso di mortalità $\mathrm{CV}$, ma in questa fascia di valori i parametri confondenti, correlabili alla mortalità, sono molto forti: età anagrafica, malnutrizione, diabete mellito ecc. In effetti la sopravvivenza dei pazienti sottoposti a paratiroidectmia totale non sembra indicare un aumento del rischio di morte, e anzi pazienti sottoposti a PTX e seguiti per 8 anni avevano un tasso di sopravvivenza migliore rispetto a quelli con PTH $>200 \mathrm{pg} / \mathrm{mL}$ (10). Anche rispetto a questo non abbiamo comunque dati definitivi, come dimostra la proposta di un trial prospettico pubblicata di recente (11).

Diverso sembrerebbe il caso dei pazienti con S-HPT più severo. In questi l'aumento della mortalità è significativo per valori molto elevati di PTH. Tuttavia l'effetto di fat- 
tori confondenti può essere tale da invertire la relazione fra PTH e mortalità. A parte fattori quali età, stato nutrizionale e altri che influenzano l'andamento sui quintili bassi del PTH, la normalizzazione per l'uso di vitamina $\mathrm{D}$ è molto importante. Nella studio di Kalanthar-Zadeh l'uso di paracacitolo si associava a un minor tasso di mortalità a parità di ogni altro fattore, ma il rischio aumentava per dosi molto elevate del farmaco (>15 $\mu \mathrm{g}$ /settimana) (3]).

Disponiamo ora dei risultati di grossi studi di intervento condotti con i farmaci di recente introdotti nella gestione dei pazienti con SHPT severo. Si tratta del già citato paracalcitolo e del cinacalcet, entrambi potenti inibitori della produzione e secrezione del PTH. L'efficienza del paracalcitolo nel ridurre i livelli di PTH è ampiamente confermata da studi controllati e prospettici in cui il farmaco è stato confrontato sia con placebo che con calcitriolo i.v. riportando riduzioni pari ad almeno il 30\% dei livelli di PTH in circa il $70 \%$ dei pazienti trattati $(12,13)$. L'associazione con cinacalcet ha consentito di far rientrare entro i target di PTH e di CaxP il 47\% dei pazienti trattati (14).

Tuttavia, rispetto agli outcome clinici le informazioni di cui disponiamo si riferiscono ad analisi retrospettive. Il noto studio di Teng attribuisce al paracalcitolo un miglioramento della sopravvivenza rispetto al calcitriolo, stimata in circa il 16\% dopo 12 mesi di trattamento, sia nei pazienti trattati in elezione con uno dei due farmaci, sia in quelli passati dall'uno all'altro (15). Il vantaggio in termini di sopravvivenza nell'uso di vitamina $\mathrm{D}$ iniettabile variava fra il $20 \%$ e il $26 \%$ in 2 anni di follow-up. Fra i pazienti in terapia rispetto a quelli non trattati vi erano 13.8 vs 28.6 decessi/ $100 \mathrm{pz} /$ anno, e le morti da causa CV erano 7.6 vs 14.6/100 pz/anno (16). Per quanto questi dati si riferiscano a una casistica multicentrica comprendente decine di migliaia di pazienti in dialisi, la loro validità è confutabile per il fatto di essere retrospettivi. Gli stessi autori concludono sulla necessità di conferma attraverso studi prospettici randomizzati.

Quanto sopra vale anche per quanto riguarda la terapia con cinacalcet. Questo farmaco riduce in modo efficace i livelli di PTH di circa il 30-40\%, in oltre il 50\% dei pazienti trattati (17), e inoltre aumenta la quota di quelli che rientrano nei target $\mathrm{K} / \mathrm{DOQI}$ per il metabolismo minerale (18). A questo è corrisposto un miglioramento degli outcome clinici in un follow-up di 6-12 mesi in cui, in particolare, il rischio di ospedalizzazione per causa $\mathrm{CV}$ si è ridotto del 39\% (19).

Anche in questo caso si trattava di un'analisi post hoc fatta su end-point secondari. Quindi anche per il cinacalcet non abbiamo certezza, sulla base di studi evidence-based, di un suo effetto sul rischio CV nei pazienti in emodialisi.

\section{Prodotto calcio fosforo}

Le considerazioni proposte per il PTH si applicano in parte al CaxP. In tutti gli studi epidemiologici già citati, il rischio $\mathrm{CV}$ correla in modo significativo con i livelli di calcemia, fosforemia e con il prodotto dei due (1-3). Il tipo di relazione calcio e fosforo ha un andamento un poco diverso. Infatti, mentre per la fosforemia è accettato un andamento ad U della curva fosforemia-mortalità, qualche dubbio esiste per la relazione con la calcemia che, secondo alcuni è di tipo monotonico (2), per altri ad $\mathrm{U}$, specie dopo correzione per le covariabili confondenti (3). Foley et al hanno per contro riportato un aumento del rischio di morte per calcemia $<8.8 \mathrm{mg} / \mathrm{dL}$ (RR 2.1), ancor più elevato per cardiopatia ischemica (RR 5.23) e per scompenso cardiaco (RR 3.3) (20). Le controversie relative al ruolo del calcio non sono marginali, in quanto coinvolgono la gestione complessiva del paziente in dialisi, inclusi la concentrazione di calcio nel bagno di dialisi, l'uso dei chelanti a base di calcio, la terapia con vitamine D. Inoltre, il rischio di ospedalizzazione per patologia $\mathrm{CV}$ non pare influenzato dai livelli di calcemia, mentre per i livelli di fosforemia più elevati il rischio aumenta dal 15 al $38 \%$ (2).

Vi è consenso unanime in letteratura sulla significativa influenza diretta del CaxP sul rischio di morte, per tutte le cause, e in particolare per causa $\mathrm{CV}$, con aumenti del 6-7\% per ogni aumento di $10 \mathrm{mg}^{2} / \mathrm{mL}^{2}$ di CaxP $(1,21)$. L'aumento del rischio è sostenuto soprattutto dalla fosforemia. Vi sono dati in letteratura che collegano un elevato CaxP con le calcificazioni coronariche e vascolari (22), e queste ultime correlano con l'aumentato rischio di morte CV $(22,23)$.

Il controllo del CaxP è in grado di influenzare la mortalità CV? Se sì, in che misura? Quali provvedimenti conviene utilizzare per perseguire questi risultati?

Sulla base dei dati epidemiologici sono oggi considerati ragionevolmente sicuri valori di fosforemia compresi fra 3.5 e $5.5 \mathrm{mg} / \mathrm{dL}$ e di prodotto $\mathrm{CaxP}<55 \mathrm{mg}^{2} / \mathrm{mL}^{2}$. E stato riportato di recente che l'aderenza per almeno 3 mesi ai target stabiliti si associa a un minor rischio di morte nei pazienti avviati alla dialisi (7). La riduzione della fosforemia ottenuta con l'uso di chelanti non contenenti calcio, in particolare con sevelamer, ha prodotto un minor incremento delle calcificazioni coronariche e aortiche (24), e una riduzione fra il 46 e $54 \%$ del rischio di ospedalizzazione (25).

Tuttavia, mentre il primo studio, prospettico, si poneva un outcome c.d. surrogato, il secondo era uno studio caso-controllo. Un recente studio prospettico multicentrico di confronto fra sevelamer e chelanti a base di calcio non è stato in grado di dimostrare differenze di 
mortalità aspecifica e da causa CV nei 1068 pazienti arruolati. Un qualche vantaggio sulla mortalità generale, ma non su quella $\mathrm{CV}$, si aveva con il sevelamer nei pazienti con età maggiore di 65 anni (26). In un recente studio di metanalisi in cui sono stati considerati 3193 pazienti arruolati in studi randomizzati, sevelamer e chelanti contenenti calcio non hanno evidenziato alcuna differenza nel tasso di mortalità, inclusa quella CV (27). Questo derivava dalla osservazione che il prodotto CaxP non differiva fra i due gruppi di trattamento.

Non disponiamo di studi prospettici e controllati relativi ai chelanti a base di calcio, nonostante questi siano in uso da moltissimi anni. A maggior ragione mancano studi centrati sull'utilizzo del carbonato di lantanio, data la sua recente immissione in commercio.

\section{Stato vitaminico $\mathbf{D}$}

La questione concernente gli effetti delle vitamine D sullo stato CV è oggetto di notevoli controversie. Da una parte gli autori che asseriscono che l'aumento del CaxP causato dalla terapia con vitamina $\mathrm{D}$ è fonte potenziale di maggior rischio $\mathrm{CV}$ per i pazienti trattati. Dall'altra quelli che sostengono che, a parità di CaxP, l'effetto di inibizione genomica della produzione di PTH e quello pleiotropico degli attivatori dei VDR è in grado di migliorare le prospettive di questi pazienti.

È indubbio che l'uso di vitamina $\mathrm{D}$ pone spesso problemi di controllo del CaxP con effetti probabilmente più rilevanti se vi è contemporaneo uso di chelanti a base di calcio. Tuttavia, gli argomenti proposti da coloro che consigliano l'uso di vitamine $\mathrm{D}$ in emodialisi sono molti, e comprendono la dimostrata potente azione di soppressione del PTH attraverso i VDR paratiroidei, soprattutto con l'uso di metaboliti attivi e con somministrazione i.v.. Inoltre, i VDR sono espressi su molti organi e tessuti, inclusi cuore e vasi, la vitamina D ha un effetto anti-infiammatorio ed antagonista sul sistema renina-angiotensina, in altre parole essa ha un effetto generale, pleiotrofico, indipendente ed aggiuntivo alla azione sul PTH. La recente introduzione di metaboliti attivi, quali il paracalcitolo (12), con minor effetto sui recettori intestinali, responsabili degli aumenti di CaxP, e maggior su quelli paratiroidei, ha tentato di rispondere all'esigenza di migliorare il profilo di sicurezza di questa classe di farmaci.

Sul piano epidemiologico abbiamo sopra ricordato due distinti studi osservazionali secondo i quali la sopravvivenza dei pazienti che hanno assunto un qualche tipo di vitamina $\mathrm{D}$ è significativamente migliore rispetto agli altri (16). Mentre fra le vitamine D iniettabili il paracalcitolo sembrava offrire un modesto ma significativo vantaggio in termini di sopravvivenza, rispetto al calcitriolo i.v. (15).
Uno studio analogo condotto sui pazienti trattati dalla Dialysis Clinic Inc. ha portato alle stesse conclusioni (28). I vantaggi sulla sopravvivenza dei pazienti non dipendono solo dall'uso dei metaboliti attivi, meglio se iniettabili, in quanto una correlazione diretta con alcuni indicatori dello stato $\mathrm{CV}$ è stata trovata non solo per il calcitriolo, ma anche per la $25(\mathrm{OH})$ vitamina $\mathrm{D}_{3}$ (29). Secondo questo studio, due indicatori di rischio $\mathrm{CV}$, la velocità dell'onda sfigmica aortica e la distensibilità dell'arteria brachiale correlavano, il primo in maniera inversa, il secondo in maniera diretta, con i livelli sierici sia di calcitriolo che di $25(\mathrm{OH}) \mathrm{D}_{3}$. L'associazione suddetta era indipendente dalle variabili confondenti, incluse età e pressione arteriosa.

Tuttavia, nonostante i molti dati sperimentali e gli studi osservazionali che evidenziano un'azione favorevole, protettiva, delle vitamine $\mathrm{D}$ sull'apparato $\mathrm{CV}$, non disponiamo ad oggi di risultati definitivi, con studi di intervento prospettici e randomizzati, da cui emerga con certezza l'efficacia dei metaboliti attivi della vitamina D nella prevenzione del danno $\mathrm{CV}$.

\section{Conclusioni}

La risposta al quesito che ci siamo posti nel titolo non appare ad oggi a portata di mano. Alcuni punti possono considerarsi acquisiti:

1) Il rischio CV è molto aumentato nei pazienti con IRC.

2) Le calcificazioni vascolari sono molto più precoci in questi pazienti, e spesso sono già presenti al momento di iniziare la terapia sostitutiva.

3) E molto probabile che le croniche persistenti alterazioni del metabolismo minerale svolgano un ruolo nella patologia $\mathrm{CV}$ del paziente in dialisi.

4) $\mathrm{Al}$ rischio $\mathrm{CV}$ concorrono tuttavia, e in modo non marginale, altri fattori tipici dell'emodializzato, quali malnutrizione, infiammazione, stress ossidativo.

Altri invece richiedono conferme acquisite con studi evidence-based:

1) La riduzione dei livelli di PTH, a parità di altri fattori confondenti, sia inerenti che estranei al metabolismo minerale, è in grado di influenzare la morbilità CV?

2) La riduzione di CaxP si traduce in una riduzione della calcificazione vascolare, ma non esistono attuali evidenze che indichino una maggiore efficacia di un chelante rispetto all'altro.

3) La più pressante indicazione all'uso della vitamina $D$ deriva dall'osservazione che in una elevata percentuale dei pazienti vi è uno stato carenziale che coinvolge sia la $25(\mathrm{OH}) \mathrm{D}_{3}$ che la $1,25(\mathrm{OH})_{2} \mathrm{D}_{3}$. I nuovi metaboliti attivi non o poco calcemizzanti sono da considerarsi al momento solo promettenti, ma attendono conferme da studi controllati. 
Questo ultimo aspetto è il più controverso. Gli studi di impatto del metabolismo minerale sugli outcome $\mathrm{CV}$ dei nostri pazienti devono essere progettati tenendo conto dei molti fattori interferenti e che sappiamo intervenire nella genesi del danno. Devono pertanto arruolare un numero elevato, ben quantizzabile, di pazienti. Deve avere una durata adeguata. Deve essere chiaro che il mancato accertamento di differenze fra due tipi di intervento, se non sostenuto da un'adeguata potenza statistica, non consente di negare con certezza che le differenze esistano. Per questo condividiamo il consenso che si è creato intorno a uno studio progettato da Amgen e avente come oggetto la valutazione dell'efficacia del cinacalcet nella prevenzione-terapia del danno CV nel dializzato (30). Lo studio intende arruolare 3800 pazienti in dialisi, da seguire per un adeguato spazio temporale, assumendo come outcome primario decessi o eventi non fatali da causa $\mathrm{CV}$, oltre alla incidenza di fratture e paratiroidectomie.

Indirizzo degli Autori:

Dr. Martino Marangella

S. C. Nefrologie e Dialisi

ASO Ordine Mauriziano

Largo Turati, 62

10128 Torino

mmarangella@mauriziano.it

\section{Bibliografia}

1. Ganesh SK, Stack AG, Levin NW, Hulbert-Shearon T, Port FK. Association of elevated serum PO (4), Ca x PO (4) product, and parathyroid hormone with cardiac mortality risk in chronic hemodialysis patients. Am Soc Nephrol 2001; 12: 2131-8.

2. Block GA, Klassen PS, Lazarus JM, Ofsthun N, Lowrie EG, Chertow GM. Mineral metabolism, mortality, and morbidity in maintenance hemodialysis. J Am Soc Nephrol 2004;15: 2208-18.

3. Kalantar-Zadeh K, Kuwae N, Regidor DL, et al. Survival predictability of time-varying indicators of bone disease in maintenance hemodialysis patients. Kidney Int 2006; 70: 771-80.

4. Go AS, Chertow GM, Fan D, McCulloch CE, Hsu CY. Chronic kidney disease and the risks of death, cardiovascular events, and hospitalization. N Engl J Med 2004; 351: 1296-305.

5. Foley RN, Parfrey PS, Sarnak MJ. Clinical epidemiology of cardiovascular disease in chronic renal disease. Am J Kidney Dis. 1998;32 (Suppl): S112-9.

6. Marco MP, Craver L, Betriu A, Belart M, Fibla J, FernÃjndez E. Higher impact of mineral metabolism on cardiovascular mortality in a European hemodialysis population. Kidney Int 2003; 85(suppl): S111-4.

7. Tentori F, Hunt WC, Rohrscheib M, et al. Which targets in clinical practice guidelines are associated with improved survival in a large dialysis organization? J Am Soc Nephrol 2007; 18: 2377-84.
8. Spiegel DM, Raggi P, Smits G, Block GA. Factors associated with mortality in patients new to haemodialysis. Nephrol Dial Transplant 2007; 21: [Epub ahead of print]

9. Neves KR, Graciolli FG, dos Reis LM, L, et al. Vascular calcification: contribution of parathyroid hormone in renal failure. Kidney Int 2007; 71: 1262-70.

10. Dussol B, Morand P, Martinat C, Lombard E, Portugal H, Brunet P, Berland Y. Influence of parathyroidectomy on mortality in hemodialysis patients: a prospective observational study. Ren Fail 2007; 29: 579-86.

11. Schlosser K, Veit JA, Witte S, et al. Comparison of total parathyroidectomy without autotransplantation and without thymectomy versus total parathyroidectomy with autotransplantation and with thymectomy for secondary hyperparathyroidism: TOPAR PILOT-Trial. Trials 2007; 8:22.

12. Martin KJ, Gonzalez EA, Gellens M, Hamm LL, Abboud H, Lindberg J. 19-Nor-1-alpha-25-dihydroxyvitamin D2 (Paricalcitol) safely and effectively reduces the levels of intact parathyroid hormone in patients on hemodialysis. J Am Soc Nephrol 1998; 9: 1427-32.

13. Sprague SM, Llach F, Amdahl M, Taccetta C, Batlle D. Paricalcitol versus calcitriol in the treatment of secondary hyperparathyroidism. Kidney Int 2003; 63: 1483-90.

14. Chertow GM, Blumenthal S, Turner S, Roppolo M, Stern L, Chi EM, Reed J; CONTROL Investigators. Cinacalcet hydrochloride (Sensipar) in hemodialysis patients on active vitamin D derivatives with controlled PTH and elevated calcium x phosphate. Clin J Am Soc Nephrol 2006; 1: 305-12.

15. Teng M, Wolf M, Lowrie E, Ofsthun N, Lazarus JM, Thad- 
hani R. Survival of patients undergoing hemodialysis with paricalcitol or calcitriol therapy. N Engl J Med 2003; 349: 446-56.

16. Teng M, Wolf M, Ofsthun MN, et al. Activated injectable vitamin $\mathrm{D}$ and hemodialysis survival: a historical cohort study. J Am Soc Nephrol 2005; 16: 1115-25.

17. Block GA, Martin KJ, de Francisco AL, et al. Cinacalcet for secondary hyperparathyroidism in patients receiving hemodialysis. N Engl J Med 2004; 350: 1516-25.

18. Moe SM, Chertow GM, Coburn JW, et al. Achieving NKF$\mathrm{K} / \mathrm{DOQI}$ bone metabolism and disease treatment goals with cinacalcet $\mathrm{HCl}$. Kidney Int 2005; 67: 760-71.

19. Cunningham J, Danese M, Olson K, Klassen P, Chertow GM. Effects of the calcimimetic cinacalcet $\mathrm{HCl}$ on cardiovascular disease, fracture, and health-related quality of life in secondary hyperparathyroidism. Kidney Int 2005; 68: 1793-800.

20. Foley RN, Parfrey PS, Harnett JD, et al. Hypocalcemia, morbidity, and mortality in end-stage renal disease. Am J Nephrol 1996; 16: 386-93.

21. Slinin Y, Foley RN, Collins AJ. Calcium, phosphorus, parathyroid hormone, and cardiovascular disease in hemodialysis patients: the USRDS waves 1 , 3, and 4 study. J Am Soc Nephrol 2005; 16: 1788-93.

22. Blacher J, Guerin AP, Pannier B, Marchais SJ, London GM. Arterial calcifications, arterial stiffness, and cardiovascular risk in end-stage renal disease. Hypertension 2001; 38: 938-42.

23. London GM, Guérin AP, Marchais SJ, Métivier F, Pannier $\mathrm{B}$, Adda H. Arterial media calcification in end-stage renal disease: impact on all-cause and cardiovascular mortality. Nephrol Dial Transplant 2003;18: 1731-40.

24. Chertow GM, Burke SK, Raggi P, Treat to Goal Working Group. Sevelamer attenuates the progression of coronary and aortic calcification in hemodialysis patients. Kidney Int 2002; 62: 245-52.

25. Collins AJ, St Peter WL, Dalleska FW, Ebben JP, Ma JZ. Hospitalization risks between Renagel phosphate binder treated and non-Renagel treated patients. Clin Nephrol 2000; 54: 334-41.

26. Suki WN, Zabaneh R, Cangiano JL, et al. Effects of sevelamer and calcium-based phosphate binders on mortality in hemodialysis patients. Kidney Int 2007; 72: 1130-7.

27. Tonelli M, Wiebe N, Culleton B, Lee H, Klarenbach S, Shrive F, Manns B; for the Alberta Kidney Disease Network. Systematic review of the clinical efficacy and safety of sevelamer in dialysis patients. Nephrol Dial Transplant 2007; 22: 2856-66.

28. Tentori F, Hunt WC, Stidley CA, Rohrscheib MR, Bedrick EJ, Meyer KB, Johnson HK, Zager PG; Medical Directors of Dialysis Clinic Inc. Mortality risk among hemodialysis patients receiving different vitamin D analogs. Kidney Int 2006; 70: 1858-65.

29. London GM, Guérin AP, Verbeke FH, et al. Mineral metabolism and arterial functions in end-stage renal disease: potential role of 25-hydroxyvitamin D deficiency. J Am Soc Nephrol 2007;18:613-20.

30. Evaluation of cinacalcet $\mathrm{HCl}$ therapy to lower cardiovascular events (Evolve) trial. ClinicalTrial.gov identifier NCT0034589. 